University of Nebraska - Lincoln

DigitalCommons@University of Nebraska - Lincoln

\title{
Exploiting the Advantages of Object-Based DSM in A Heterogeneous Cluster Environment
}

\author{
Xuli Liu \\ University of Nebraska-Lincoln, xuliu@cse.unl.edu \\ Hong Jiang \\ University of Nebraska-Lincoln, jiang@cse.unl.edu \\ Leen-Kiat Soh \\ University of Nebraska, Isoh2@unl.edu
}

Follow this and additional works at: https://digitalcommons.unl.edu/cseconfwork

Part of the Computer Sciences Commons

Liu, Xuli; Jiang, Hong; and Soh, Leen-Kiat, "Exploiting the Advantages of Object-Based DSM in A Heterogeneous Cluster Environment" (2005). CSE Conference and Workshop Papers. 17.

https://digitalcommons.unl.edu/cseconfwork/17

This Article is brought to you for free and open access by the Computer Science and Engineering, Department of at DigitalCommons@University of Nebraska - Lincoln. It has been accepted for inclusion in CSE Conference and Workshop Papers by an authorized administrator of DigitalCommons@University of Nebraska - Lincoln. 


\title{
Exploiting the Advantages of Object-Based DSM in A Heterogeneous Cluster Environment
}

\author{
Xuli Liu, Hong Jiang, Leen-Kiat Soh \\ Department of Computer Science and Engineering, University of Nebraska-Lincoln \\ \{xuliu, jiang, lksoh\}@cse.unl.edu
}

\begin{abstract}
In recent years, increasing effort has been made by the cluster and grid computing community to build object-based Distributed Shared Memory systems (DSM) in a cluster environment. In most of these systems, a shared object is simply used as a data-exchanging unit so as to alleviate the false-sharing problem, and the advantages of sharing objects remain to be fully exploited. Thus, this paper is motivated to investigate the potential advantages of object-based DSM. For example, the performance of a distributed application may be significantly improved by adaptively and judiciously setting the size of the sharedobjects, i.e., granularity. This paper, in addition to investigating the advantages of sharing objects, particularly focuses on observing how the performance of a distributed application changes with varied granularity, obtaining the optimal granularity through curve fitting, studying the factors that affect the optimal granularity, and predicting this optimal granularity in a changing runtime environment.
\end{abstract}

\section{Introduction}

Parallel/distributed programming has two prevailing communication abstractions-explicit Message Passing (MP) and Distributed Shared Memory (DSM). Due to its programming complexity, MP has been replaced with DSM on multiprocessor computers, such as DASH [7] at Stanford, Alewife [2] at MIT, TreadMarks [6] at Rice, IVY [9] at Yale, etc. Nevertheless, MP remains the dominant programming paradigm on cluster computers. The reason is that the design of DSM on multiprocessor computers cannot be transplanted easily and directly onto cluster computers due to the significant difference in the communication infrastructure between the tightly coupled multiprocessor systems and the loosely coupled cluster systems. For example, compared to multiprocessor computers, cluster computers have much higher communication latency and lack hardware support for replication and consistency control. This provides impetus for researchers to redesign DSM on cluster computers to overcome the associated problems.

The design of DSM on multiprocessor computers is based on either cache lines or memory pages. Although some of the DSM systems on cluster computers are still page-based, e.g., Java/DSM [19], most of them provide object-based DSM, such as JavaParty [13], Jackal [15], Hyperion [12], and cJVM [1]. To the best of our knowledge, most of the current studies on object-based DSM are still in the phase of building research platforms and solving the related implementation issues. In these systems, an object is simply used as a data-exchanging unit similar to a cache line or a memory page in the DSM of multiprocessor computers. Nevertheless, some key studies have been carried out, aiming at improving the efficiency of the object-based DSM. For example, Chang et al. [3] proposed a mechanism to assign different consistency levels appropriate for different shared objects for interactive applications on wide area distributed systems. However, the aforementioned studies focus on either the implementation issues or improving the efficiency of the DSM from some specific aspects such as communication latency and bandwidth requirements. Thus, these studies fall short of systematically addressing the advantages of sharing objects, or attempting to fully exploiting such advantages.

In this paper, in addition to investigating the advantages of sharing objects, we focus on the shared-object size (i.e., granularity) problem. We run the LU benchmark application [18] using various granularities, combined with varied runtime factors. By observing the performance variations, we conclude that an optimal granularity can be obtained through curve fitting. Furthermore, we empirically derive an equation to predict this optimal granularity in a changing runtime environment. We also observed important LU-related properties on clusters, such as the insensitivity of problem size and network bandwidth to the determination of optimal granularity.

The rest of this paper is organized as follows. In Section 2, existing studies in the literature on object-based DSM systems are briefly reviewed. Section 3 studies the 
advantages of object-based DSM systems over traditional DSM systems. In Section 4, we briefly introduce our object-based DSM prototype system called PJava [11]. The methodology for obtaining the optimal granularity is presented in Section 5. Finally, we conclude and outline our future work in Section 6.

\section{Related work}

Due to the portability of Java as well as its parallel features such as Remote Method Invocation (RMI), socket, monitor, and multithread, most of the current DSM studies on cluster computers concentrate on distributed Java Virtual Machine (JVM). Three approaches are available to construct distributed JVM. The first one is to lay the distributed JVM on top of some existing DSM system. For example, Java/DSM [19], built on top of the page-based DSM of TreadMarks [6], provides a virtual shared memory Java programming environment. Unfortunately, Java/DSM inherits the page-based feature from TreadMarks, which is incompatible with the object-based memory model of Java and that prevents Java/DSM from exploiting the advantages of Java objects.

The second approach is based on a cluster-enabled implementation of JVM, of which cJVM [1] is an example. This implementation approach is able to support any pure Java application without requiring any code modification; however, it suffers from the difficulty in keeping up with the evolution of JVM. In cJVM, each node runs a cJVM process to execute part of the application's Java threads and contains a portion of the Java objects.

The third approach, which is the most widely used by current research projects, is to put another layer on top of JVM and provide the necessary parallel and distributed features. For example, JavaParty [13] provides a virtual object-based shared address space by translating JavaParty code into pure Java code with RMI running on JVM. Hyperion [12] supports parallel execution by distributing Java threads across multiple nodes in a cluster computer and providing an object-based virtual shared memory. Furthermore, in order to speed up the parallel execution, Hyperion provides a Java-bytecode-to-C translator. Jackal [15] provides a fine-grained DSM, which is managed as a collection of regions distributed on multiple cluster nodes. Each region is either an object or a fixedsize partition of an array. PJava [11], an ongoing project by the authors of this paper, provides programmers with a virtual object-based DSM by translating the PJava code into pure Java code and handling the communication among cluster nodes via sockets.

Besides distributed JVM, distributed DSM has been implemented on other platforms. For instance, Seidmann [14] presented a Distributed DSM system as an extension to the Microsoft .NET framework.
Some studies with more focus on improving the performance of distributed DSM systems have been carried out and reported in the literature. With an excessive number of replicas in a system, the benefits obtained from increased locality can be offset by the cost of maintaining consistency among the replicas. Leonardo et al. [8] proposed an adaptive scheme to determine on the fly the number of replicas to be made and the locations to store these replicas. In the distributed JVM system presented by Fang et al. [4], object migration, thread migration, and object pre-fetching are adopted to improve the performance. In order to alleviate contention on the lock applied to a distributed shared object, Wong et al. [17] presented a multi-locking mechanism in an object-based DSM system called Jasmine [16]. In Jasmine, multiple locks are applied to different data sets of a shared object to enhance the access concurrency.

\section{The advantages of sharing objects}

Some object-based DSM systems have been built and studied. However, these studies have not systematically addressed the advantages of using shared objects in DSM and investigated ways to fully exploit such advantages. This section identifies several such advantages.

First, the data-exchanging unit in a traditional DSM system is either a cache line or a memory page. Therefore, either the problem of insufficient locality due to the small cache line or the problem of false-sharing due to the large page size becomes inevitable, potentially degrading the performance of distributed applications significantly. On the other hand, an object-based DSM system only encapsulates related data into one single object, which effectively alleviates the false-sharing problem while capturing sufficient amount of locality. Furthermore, the size of a shared object could be judiciously adapted to benefit the performance of distributed applications.

Second, the object-oriented programming paradigm, in which the memory is managed based on objects, is widely used in practice by programmers. Thus, objectbased DSM can better support the object-oriented programming.

Third, it is important to notice that only related data is encapsulated into one single object. Therefore, the data in one shared object may exhibit very similar access patterns; meanwhile, different shared objects may have different access patterns. Based on these access patterns, varied coherence and/or consistency protocols may be applied to different shared objects. For example, an update-based coherence protocol is suitable for a shared object that is frequently referenced by many but relatively infrequently modified; whereas, a frequently modified but infrequently referenced or shared object prefers an invalidation-based coherence protocol. 
Finally, replication is widely used in DSM systems to improve locality, and a corresponding mechanism is required to maintain the consistency among replicas. Based on the fact that different shared objects may have varied requirements on the Quality of Consistency $(\mathrm{QoC})$, it is possible to assign different consistency levels to different shared objects, so as to improve the performance of the distributed application. QoC allows the level of consistency of a shared object to be specified and monitored in our compilation environment, which will be further discussed in Section 4.1.

\section{PJava}

Instead of building a distributed JVM from scratch, PJava is built on top of JVM, providing users with an object-based distributed JVM. In this section, the compilation and runtime environments of PJava are briefly introduced, and an in-depth description could be found in [11].

\subsection{The PJava compilation environment}

In PJava, the standard Java language is extended to support parallel programming for distributed applications. Through this programming interface, users are able to use compilation directives to specify how to parallelize a task and partition the data, and to define globally shared objects and local objects, etc. Figure 1 shows a block diagram of the compilation environment of PJava.

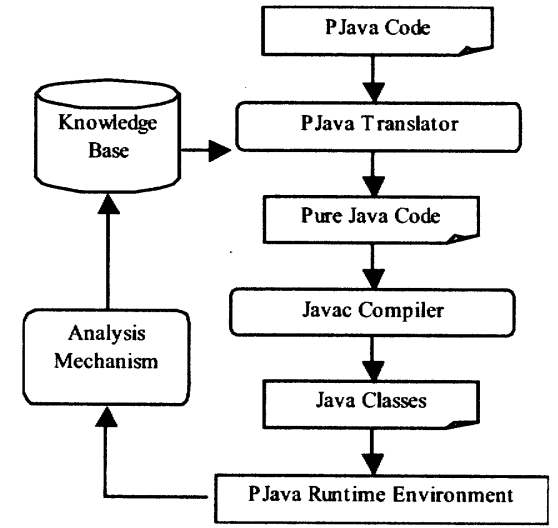

\section{Figure 1. The compilation environment of PJava}

From Figure 1, we see that the PJava translator takes the PJava code as input, parallelizes the task and partitions the data, and outputs the pure Java code. During this process, heuristics stored in the knowledge base are referenced so as to determine the proper sharing level and consistency protocol for each shared object, and to choose an optimal shared-object size, and so on.

The information stored in the knowledge base is used to support the translation described above. It includes (1) the access pattern for each shared object, which is used to choose a proper coherence and/or consistency protocol for that object; (2) the consistency level assignment for each shared object, which is determined by the QoC requirements defined by the user; (3) the optimal sharedobject size, which is used to guide the data partition process of a parallel task; and (4) the runtime environment factors, e.g., CPU usage and network bandwidth availability. The data in the knowledge base is initially entered and updated by the users, but will later be automatically adjusted based on the feedback obtained from the analysis mechanism. The QoC requirements include factors such as numerical error tolerance level, latency requirement, staleness tolerance level, synchronization requirement, etc. The pure Java code generated by the PJava translator is then compiled by the standard Javac compiler and is executed on the PJava runtime environment. At the same time, the execution information, e.g., the access patterns of each shared object and the CPU usage, is collected and analyzed by the analysis mechanism, and the analysis result is fed back to the knowledge base to adjust and/or update the related information.

In the current phase of this study, most of the components shown in Figure 1 have been implemented and this PJava compilation environment is operational. However, the knowledge base does not exist yet, only a few modules of the analysis mechanism are implemented, and the parallelization of tasks is not fully automated. In the experiments conducted in this paper, the runtime information, e.g., CPU and network bandwidth availabilities, is manually collected and analyzed, and the knowledge is fed into the PJava translator by hand.

The innovation of our PJava compilation environment lies with its use of knowledge base and embedded analysis mechanism; this is advantageous because it adaptively adjusts to optimize the parallelization and resource allocation strategies according to the dynamics of the system and characteristics of the application.

\subsection{The PJava runtime environment}

Figure 2 shows the fully implemented runtime environment of PJava on the logical head node and on one of the many work nodes. Note that the runtime environment on all work nodes is logically identical. The logical head node accepts from a user the PJava code, generates the corresponding standard Java classes and partitions the data using the heuristics in the knowledge base, and then maps the Java classes and data onto the work nodes. The logical head node is also responsible for returning the execution result to the user. The reason for using a logical head node instead of a fixed physical one is to avoid the single point of failure and the possible bottleneck.

On each work node, a daemon process keeps watching for incoming tasks and data. After getting a task, a corresponding work thread is created to execute this task, 
and necessary service threads are spawned to handle the communication with other work nodes. In this objectbased DSM environment, a table is maintained on each node to manage the shared objects. Besides the data, a shared object also includes other status information including the object state, the sharing level, the location of the up-to-date copy, etc. Replication is supported in PJava to enhance locality. The Distributed Shared Object Supporting Package (DSO-SP), built on top of the JVM, provides the necessary functionalities to maintain the consistency among replicas [10]. After the task is completed, the execution result is returned to the logical head node, along with such execution information as CPU usage, etc.

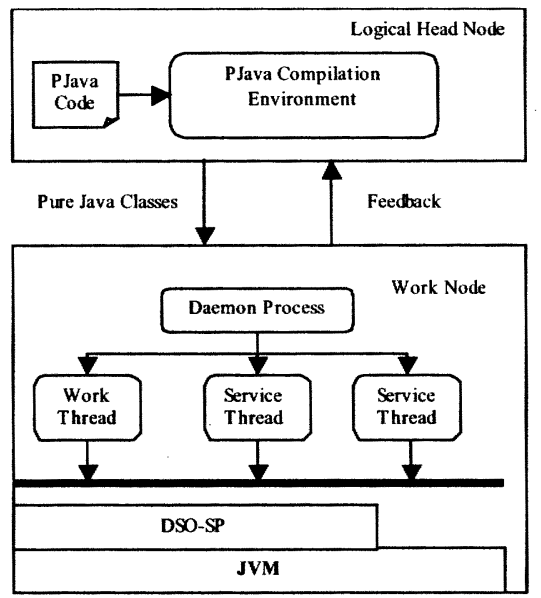

Figure 2. The runtime environment of PJava

\section{Determining the optimal shared-object size (granularity)}

In Section 3, some important advantages of an object-based DSM system are identified. In this section, we focus on one of these advantages - the issue of determining an optimal shared-object size, or object granularity. In a traditional cache-line-based or page-based DSM system, it is not easy to switch between cache-line granularity and memory-page granularity, let alone flexibly and adaptively setting the size of data-exchanging unit. On the other hand, an object-based DSM system is flexible in that regard. This motivates us to investigate ways to determine the optimal shared-object size for data-parallel applications in this paper.

For our investigation, we study the performance of the benchmark application LU [18] with varied sharedobject sizes. LU, which factors a dense matrix into the product of a lower triangular matrix and an upper triangular matrix, lies at the heart of many scientific and engineering applications. We observe that an optimal sharedobject size does exist and is affected by several runtime environment factors. Based on the observation, we find out that a formula could further be obtained through curve fitting to predict this optimal shared-object size under changing runtime environments. Note that what we propose here is a methodology, and through our experiments, show how we conduct our search for a formula that adapts to the environmental factors for the benchmark application LU.

In what follows, we first describe the experimental setup, and then present a theoretical analysis on the relationship between the application performance and the shared-object size. The methodology starts with the observation of the performance variance of $\mathrm{LU}$ at different shared-object sizes. We then investigate the factors affecting the optimal shared-object size. Subsequently, curve fitting is used to identify the optimal shared-object size and a formula is derived. Finally, we validate the formula through further testing.

\subsection{Experimental Setup}

The experiment is carried out using the benchmark application LU from the SPLASH-2 suite. The original benchmark program was in c language, and we rewrote it using Java to make it object-oriented. LU factors a dense matrix into the product of a lower triangular and an upper triangular matrix. Here, the parallel algorithm is briefly described, and more details could be found in [18].

Assuming that the total number of work nodes is $p$ and the size of the matrix to be factorized is $n \times n$, the matrix is partitioned into a set of sub-matrices with size $m \times m$ (i.e., the so-called granularity), and these sub-matrices are mapped to the $p$ work nodes using a 2-D scatter decomposition. Starting from the top-left sub-matrix, the sub-matrices are LUed by its owner along the diagonal of the matrix downwards to the bottom-right. After a diagonal sub-matrix is LUed, all other sub-matrices on its bottom-right starts being processed at once, after which the next diagonal sub-matrix will be dealt with until all of the diagonal ones are processed. Obviously, synchronization exists among the work nodes.

The experimental platform is the Prairiefire Supercomputer at University of Nebraska-Lincoln, a highperformance cluster computer with 128 nodes. Each node has two AMD 2.2 G Opteron (64-bit) processors and 4GB RAM, and the nodes are connected by a Myrinet and a Gigabit Ethernet.

\subsection{Theoretical Analysis}

Roughly speaking, the total running time of a distributed application consists of three parts, namely, the computation time, communication time, and synchronization time, as shown in Equation (1).

$$
T_{\text {total }}=T_{\text {comp }}+T_{\text {comm }}+T_{\text {sync }}
$$


Intuitively, given a distributed application and an object-based DSM platform, $T_{\text {total }}$ is affected by the sharedobject size (i.e., granularity), the problem size, the number of work nodes, the CPU availability, the networkbandwidth availability, and the characteristics of the application such as the parallel algorithm and synchronization. Conceptually, this relationship can be expressed as in the following equation:

$$
T_{\text {total }}=f_{\text {total }}(g, s, n, c, b, p)
$$

In Equation (2), $g$ stands for the shared object size (i.e., granularity), $s$ symbolizes the problem size, $n$ denotes the number of work nodes, $c$ is the CPU availability, $b$ signifies the network-bandwidth availability, and $p$ describes the application characteristics such as synchronization behavior and parallelism level.

The goal of this research is to study how the application performance is affected by $g$, and to find the optimal $g$ so as to minimize $T_{\text {total }}$ of a distributed application. Using $g *$ to express the optimal $g$, we have

$$
g^{*}=\underset{s, n, c, b, p}{\arg \min }\left(T_{\text {total }}\right)
$$

Equation (3) means that $\mathrm{g}^{*}$ is the value where $T_{\text {total }}$ is the minimum value under the given set of $s, n, c, b$, and $p$. It is obvious that $g^{*}$ is dependent on $s, n, c, b$, and $p$, i.e.,

$$
g^{*}=f_{g}(s, n, c, b, p)
$$

In the following, we study how to obtain and adapt $g^{*}$ in a changing runtime environment. Note that since $p$ is an inherent property of an application, it is omitted from the current phase of this research.

\subsection{Obtaining $g *$ through curve fitting}

After observing the performance data of LU with different matrix sizes $(s)$ and different numbers of work nodes $(\vec{r})$ in a dynamic environment, we conclude that the application performance is highly affected by $g$, and the optimal $g$, i.e., $g^{*}$, exists. As an example, Table 1 shows the data obtained when factoring a $3500 \times 3500$ matrix on 12 work nodes with full CPU and network availability. Other experiments on different matrix sizes with different number of work nodes under various system workloads had been undertaken and showed similar effects.

Table1. Execution time of LU on 12 work nodes with varied granularity

\begin{tabular}{|c|c|}
\hline Granularity & Execution Time (Seconds) \\
\hline $100 \times 100$ & 229.700 \\
\hline $150 \times 150$ & 130.766 \\
\hline $200 \times 200$ & 105.513 \\
\hline $250 \times 250$ & 71.900 \\
\hline $300 \times 300$ & 62.051 \\
\hline $350 \times 350$ & 65.525 \\
\hline $400 \times 400$ & 100.509 \\
\hline
\end{tabular}

The data in Table 1 is shown in Figure 3 as a curve that can be expressed with the formula in Equation (5):

$$
f(x)=586.6 e^{-0.009695 x}+1.309 e^{0.01047 x}
$$

Let $d f(x) / d x=0$, and we obtain the optimal value for $x=299$. Setting $g=299 \times 299$, we run the LU application with the same settings as those in Table 1 , and we get $T_{\text {total }}=58.458$ (Seconds), which is less than all the sample $T_{\text {total }}$ values shown in Table 1. This hints that the mathematical equation (i.e., Eq. 5) can be used to obtain the optimal granularity size that can be validated by the actual run on the benchmark application of LU.

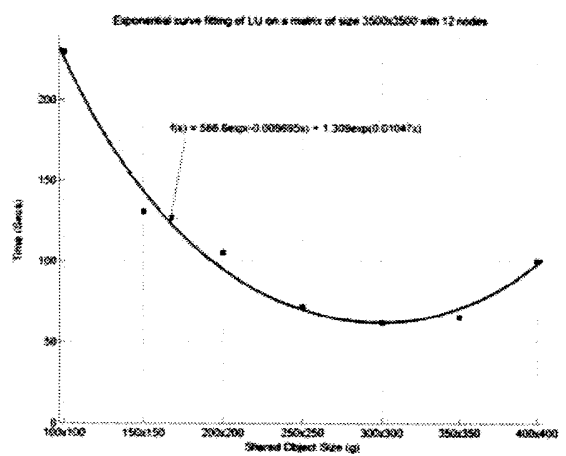

Figure 3. Exponential curve fitting of data in Table 1

\subsection{Impacts of $s, n, c$ and $b$ on $g$ *}

In this subsection, we study the impacts of $s, n, c$ and $b$ on $g^{*}$. First, given full CPU and network bandwidth availability, the changes of $g^{*}$ is evaluated with multiple matrix sizes $(s)$ running on different number of work nodes $(n)$. And then, we evaluate the variation of $g^{*}$ after taking away some CPU power $(c)$ and network bandwidth (b).

5.4.1. The impacts of $\boldsymbol{s}$ and $\boldsymbol{n}$ on $\boldsymbol{g}$ *. Varying $g$, we run LU on different numbers of work nodes $(n)$ with different matrix sizes $(s)$. As an example, Figure 4 shows the performance data of $\mathrm{LU}$ with different matrix sizes $(2500 \times 2500,3000 \times 3000,3500 \times 3500$, and $4000 \times 4000)$, each with 4 work nodes. Correspondingly, Figure 5 shows the results of the experiment with 12 work nodes. The curves shown in these two figures are obtained by applying shape-preserving interpolation [5] on the sample data. Both Figures 4 and 5 show that

- $T_{\text {comp }}$ increases with $g$. The reason is that a larger $g$ causes more cache misses, which leads to more computation time.

- A smaller $g$ implies a bigger number of shared objects and more frequent communication. Thus, $T_{\text {comm }}$ decreases as $g$ increases.

- As $g$ increases, $T_{s y n c}$ first decreases and then increases after $g$ reaches some value. The reason is that when $g$ is small, a large number of synchronization activities are 
needed; on the other extreme, when $g$ gets too large, each synchronization operation takes too long. Therefore, an optimal shared-object size exists for $T_{s y n c}$, and this value is based on the application characteristics.

- Based on the observed characteristics of $T_{\text {comp }}, T_{\text {comm }}$, and $T_{\text {sync, }}, g^{*}$ exists for $T_{\text {total }}$ though it is not very clear.

- $g^{*}$ is not quite sensitive to $s$ (i.e., the matrix size in LU). Therefore, $g^{*}$ needs not be adjusted when $s$ changes.
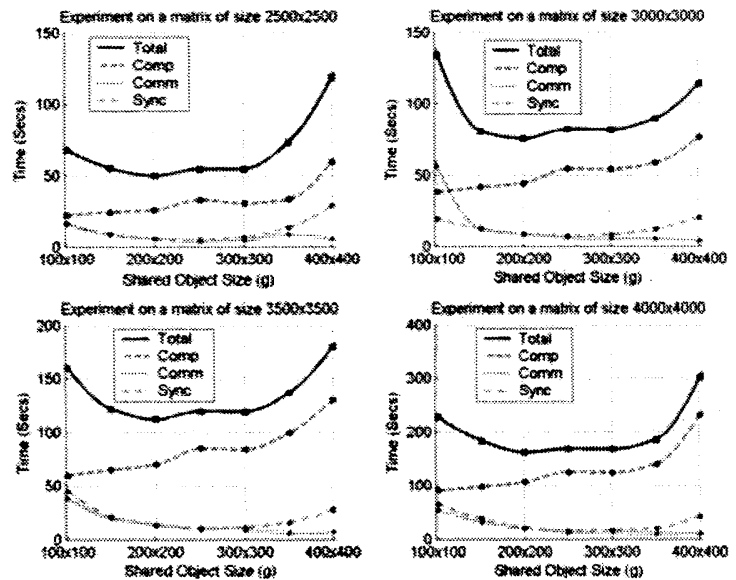

Figure 4. Performance evaluation on 4 work nodes
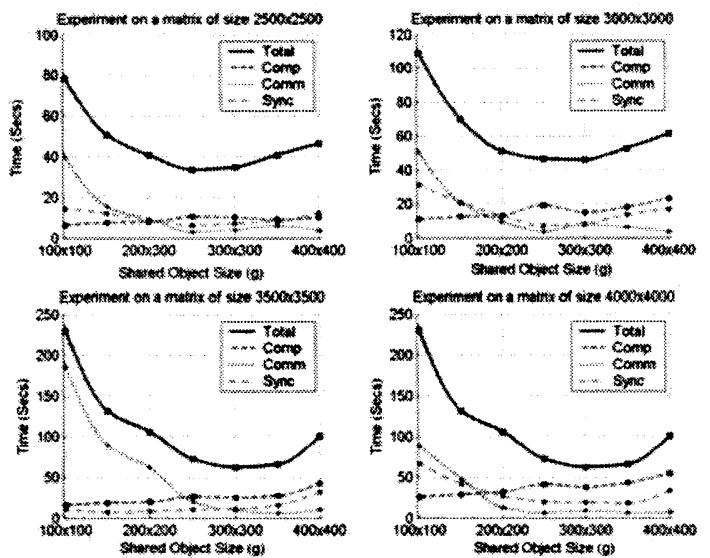

Figure 5. Performance evaluation on 12 work nodes

By comparing Figures 4 and 5, we see that $n$ affects $g^{*}: g^{*}$ is around $200 \times 200$ in the 4-node case and about $300 \times 300$ in the 12 -node case. In the 4-node case, $T_{\text {comp }}$ is the dominant component of $T_{\text {total }}$, so a smaller $g$ is preferred. On the other hand, $T_{\text {comm }}$ starts to play a more important role in the 12-node case, thus a larger $g$ is more appropriate. Other experiments on different number of work nodes with varied matrix sizes demonstrated comparable results.

5.4.2. The impact of $c$ on $\boldsymbol{g}^{*}$. With $60 \%$ of $c$ left (i.e., by running another application taking $40 \%$ of $\mathrm{CPU}$ ), we run the $L U$ application with varied matrix sizes on different total numbers of work nodes, and observe how the execution time varies compared to the data obtained with $100 \%$ $c$ availability. As an example, Figure 6 shows this comparison on a matrix of size $4000 \times 4000$ with 8 nodes.

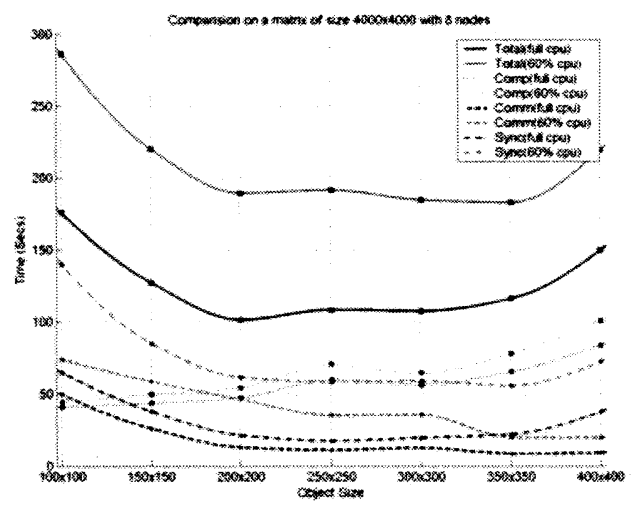

Figure 6. Comparison on a matrix of size $4000 \times 4000$ with 8 work nodes $(100 \%$ vs. $60 \%$ CPU availability)

From Figure 6, we see that $T_{\text {total }}, T_{\text {comp }}, T_{\text {comm }}$, and $T_{s y n c}$ all increase with a reduced $c$. It is easy to understand the increase in $T_{\text {comp }}$. The increase in $T_{\text {sync }}$ is due to the increased waiting time for shared objects that now take longer time to compute. It is important to notice that, despite the increase in $T_{\text {comp }}$ and $T_{\text {sync }}$, the shapes and patterns of their curves remain almost identical to the curves with $100 \% c$ availability.

Figure 6 shows that $T_{\text {comm }}$ increases too. The reason is that $\mathrm{CPU}$ is involved in building the socket connections in communication. Therefore, $T_{\text {comm }}$ increases when less CPU processing power is available. What is more important is that the shape of the communication time curve changes after some CPU power is taken awaysuggesting that a larger $g$ is preferred since a larger $g$ causes less frequent communication. As a result, $g^{*}$ varies from about $200 \times 200$ (with $100 \% c$ availability) to around $350 \times 350$ (with $60 \% c$ availability). Thus, we conclude that $g^{*}$ increases as the CPU contention intensifies, and this change comes solely from the fluctuation in the communication time. Other experiments on different matrix sizes and varied number of work nodes showed the same result.

5.4.3. The impact of $\boldsymbol{b}$ on $\boldsymbol{g}$ *. We perform a similar experiment as in Section 5.4 .2 by taking away $40 \%$ of the network bandwidth, and the result is shown in Figure 7.

From Figure 7, we can see that $T_{\text {total }}, T_{\text {comp }}$, and $T_{\text {sync }}$ all increase slightly. The reason is that the program used for consuming the network bandwidth uses a small amount of CPU processing power. As a cluster computer always uses a high-speed network to connect the nodes (e.g., a Myrinet and a Gigabyte Ethernet are used in Prai- 
riefire), the contention on the network bandwidth does not noticeably slow down the communication in distributed applications. Actually, the time to set up the socket connections dominates the total communication time if the data size is not too large in each transmission. As a result, $T_{\text {comm }}$ remains almost unchanged and $g^{*}$ remains unchanged as the contention on the network bandwidth gets intense, as shown in Figure 7. Therefore, we conclude that the impact of $b$ on $g^{*}$ can be neglected. Similar experiments on other matrix sizes with different number of work nodes under varied network workloads also claimed the insensitivity of $g^{*}$ to the variation of $b$.

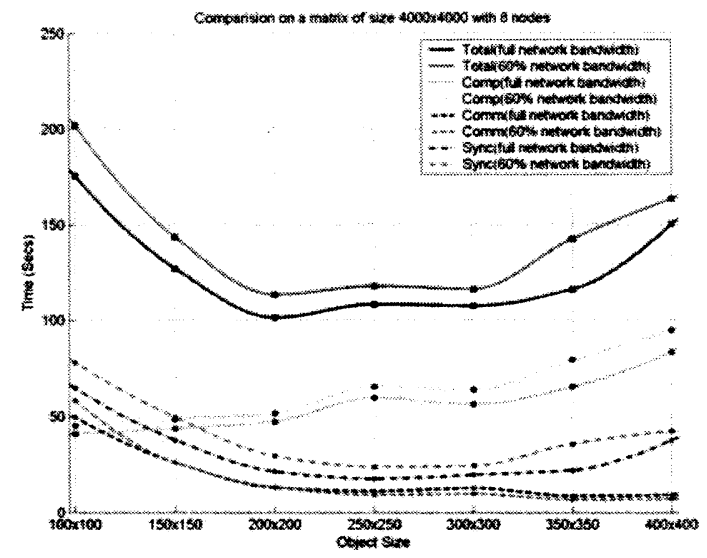

Figure 7. Comparison on a matrix of size $4000 \times 4000$ with 8 nodes (100\% vs. $60 \%$ network bandwidth availability)

\subsection{Adapting $g *$ to varying $c$ for a given $n$}

In most cluster environments, a user submits his/her application to the system scheduler by specifying the number of work nodes, $n$, required by the application, among other parameters. In order to figure out a solution to adjust $g^{*}$ with varying $c$, for a given $n$, we carry out a set of experiments to observe the changes of $g^{*}$ with varied $c$ values. As an example, Table 2 contains the experimental results on a matrix of size $3500 \times 3500$ with 4 work nodes. Note that $g^{*}$ in Table 2 is obtained through the curve fitting described in Section 5.3.

\section{Table 2. Variation of $g^{*}$ with $\mathrm{c}$ on a matrix of size $3500 \times 3500$ with 4 work nodes}

\begin{tabular}{|c|c|c|c|c|}
\hline CPU availability & $100 \%$ & $90 \%$ & $80 \%$ & $70 \%$ \\
\hline g* & 227 & 237 & 248 & 261 \\
\hline
\end{tabular}

We apply exponential curve fitting to the data in Table 2, and show the result in Figure 8. The curve shown in Figure 8 is expressed by a formula as in Equation (6):

$$
f(x)=226.8 \times e^{0.004165 x}+0.2124 \times e^{0.09806 x}
$$

Using Equation (6), we predict $g^{*}$ by taking away $18 \%, 25 \%$, and $38 \%$ of CPU processing power, and compare the predicted values to the real values. The comparison results are listed in Table 3, and the real values are also shown by circle-markers in Figure 8 .

Based on the results shown in Table 3, we speculate that Equation (6) could be used to predict $g^{*}$ accurately. Other experiments also showed the predicitability of $g^{*}$ with varied $c$.

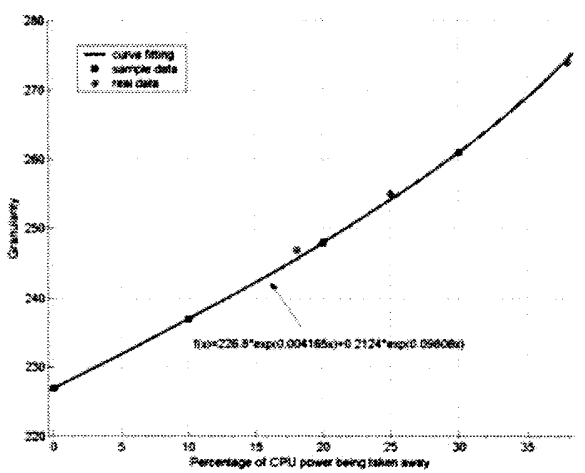

Figure 8. Exponential curve fitting of the data in Table 2

Table 3. Comparison of predicted $\mathbf{g}^{*}$ and real $\mathrm{g}^{*}$

\begin{tabular}{|c|c|c|}
\hline $\begin{array}{c}\text { Percentage of CPU } \\
\text { power being taken away }\end{array}$ & $\begin{array}{c}\text { Predicted } \\
\text { value }\end{array}$ & Real value \\
\hline $18 \%$ & 246 & 247 \\
\hline $25 \%$ & 254 & 255 \\
\hline $38 \%$ & 275 & 274 \\
\hline
\end{tabular}

\subsection{Methodology}

Summarizing the above observations, we conclude that $g^{*}$ is sensitive to $n$ and $c$, and insensitive to $s$ and $b$. That is, when the problem size $(s)$ and network bandwidth availability $(b)$ change, $g^{*}$ does not need to be adjusted. When the total number of work nodes $(n)$ changes, it is necessary and reasonable to obtain $g^{*}$ again through curve fitting since $n$ is usually an input parameter by the user. Given a distributed application and fixed number of work nodes, by a user for the very first time, it is worthwhile to collect some sample data and perform a curve fitting as done in Section 5.5 to obtain a formula like Equation (6) so as to predict $g^{*}$ whenever $c$ changes. This predictive formula can then be stored in the knowledge base of Figure 1 for optimizing performances of future submissions of similar applications. We re-calculate $g^{*}$ each time we re-run a distributed application. For some applications such as online collaborative applications and online gaming, it is possible to adjust $g^{*}$ on the fly.

\section{Conclusion and Future work}


In this paper, we have systematically investigated the advantages of object-based DSM systems, and especially focused on how to adaptively set the optimal sharedobject size for a distributed application. Based on our experiments using the benchmark application LU, we have concluded that the optimal shared object size $g^{*}$ can be obtained through curve fitting. We have also observed that $g^{*}$ is highly sensitive to the total number of work nodes and CPU contention; whereas, $g^{*}$ is not quite sensitive to the problem size and network bandwidth contention. We have also speculated that a formula obtained through curve fitting could be used to predict $g^{*}$ in a dynamic runtime environment.

The methodology proposed in this paper had only been evaluated by one test case, i.e., the SPLASH LU benchmark, and its extent of generality remains to be established. In the future, more experiments need to be carried out on other test cases so as to observe whether comparable results could be achieved. Furthermore, we will implement the adaptive mechanism, completing the analysis component that is part of the overall approach (Figure 1). Moreover, we will investigate in details other advantages outlined in Section 3. In addition to supporting scientific applications, we plan to adapt the infrastructure of PJava to support on-line collaboration applications and collaborative computing.

\section{Acknowledgement}

This work was supported in part by a seed grant from the National Center for Information Technology in Education, a NSF SBIR Grant (DMI-0441249), and a Chinese NSF 973 Project Grant (2004cb318201). Research Computing Facility (RCF) at University of Nebraska-Lincoln provided the experimental environment-Prairiefire $s u$ percomputer, and Dr. David Swanson and Makoto Furukawa kindly supported the experiments. The authors would like to thank Yifeng Zhu and the anonymous reviewers for their comments to this work.

\section{References}

[1] Y. Aridor, M. Factor and A. Teperman, "cJVM: a Single System Image of a JVM on a Cluster", Proc. 1999 Int. Conf. on Parallel Processing, Fukushima, Japan, September 1999, pp. 411.

[2] D. Chaiken, J. Kubiatowicz, and A. Agarwal, "LimitLESS Directories: A Scalable Cache Coherence Scheme", Proc. $4^{\text {th }}$ Int. Conf. on Architectural Support for programming Languages and Operating System", NY, April 1991, pp. 224-234.

[3] T. Chang, G. Popescu, and C. Codella, "Scalable and Efficient Update Dissemination for Distributed Interactive Applications”, Proc. $22^{\text {nd }}$ Int. Conf. on Distributed Computing Systems, Vienna, Austria, July 2002, pp. 143-152.

[4] W. Fang, C. Wang, and F. Lau, "Efficient Global Object Space Support for Distributed JVM on Cluster", Proc. 2002 Int.
Conf. on Parallel Processing, Vancouver, Canada, August 2002, pp. 371-378.

[5] T. N. T. Goodman, "Shape Preserving Interpolation by Curves", Algorithms for Approximation IV, J. Levesley, I.J. Anderson, J.C. Mason (eds.), University of Huddersfield, 2002, pp.24-35.

[6] P. Keleher, S. Dwarkadas, A. L. Cox, and W. Zwaenepoel, "Treadmarks: Distributed Shared Memory on Standard Workstations and Operating Systems", Proc. 1994 Winter Usenix Conf., San Francisco, California, USA, January 1994, pp. 115-131.

[7] D. Lenoski, J. Laudon, K. Gharachorloo, A. Gupta, and J. hennessy, "The Directory-Based Cache Coherence Protocol for the DASH Multiprocessor", Proc. $17^{\text {th }}$ Annual Int. Symp. on Computer Architecture, Seattle, WA, June 1990, pp. 148-159.

[8] J. C. Leonardo, T. Takaichi and S. Narazaki, "Fault Tolerant Shared-Object Management System With Dynamic Replication Control Strategy", Proc. $7^{\text {th }}$ Int. Conf. on Parallel and Distributed Systems, Iwate, Japan, July 2000, pp. 524-529.

[9] K. Li and P. Hudak, "Memory Coherence in Shared Virtual Memory Systems", ACM Transactions on Computer Systems, Volume 7, Issue 4, November 1989, pp. 321-359.

[10] X. Liu, H. Jiang, and L. K. Soh, "A Distributed Shared Object Model Based on Hierarchical Consistency Protocol for Heterogeneous Clusters", Proc. $4^{\text {th }}$ IEEE/ACM Int. Symp. on Cluster Computing and the Grid, Chicago, IL, April 2004, pp. 515-522.

[11] X. Liu, H. Jiang, and L. K. Soh, "PJava: A Distributed Environment on Top of JVM", Technical Report TR04-11-01, CSE, University of Nebraska-Lincoln.

[12] M. W. MacBeth, K. A. McGuigan and P. J. Hatcher, "Executing Java Threads in Parallel in a Distributed-Memory Environment", Proc. $8^{\text {th }}$ Annual IBM Centers for Advanced Studies Conf., Missisauga, Ontario, Canada, December 1998, pp. 40-54. [13] M. Philippsen and M. Zenger, "JavaParty - Transparent Remote Objects in Java", Concurrency: Practice and Experience, Volume 9, Number 11, November 1997, pp. 1225-1242.

[14] T. Seidmann, "Distributed Shared Memory Using The .NET Framework", Proc. $3^{\text {rd }}$ IEEE/ACM Int. Symp. on Cluster Computing and the Grid, Tokyo, Japan, May 2003, pp. 457-462. [15] R. Veldema, R. A. F. Bhoedjang and H. E. Bal, "Distributed Shared Memory Management for Java", Proc. $4^{\text {th }}$ Annual Conf. of the Advanced School for Computing and Imaging, Lommel, Belgium, June 2000, pp. 256-264.

[16] A. K. L. Wong and W. Zhu, "An Implementation of Pure Java Based DSM", Proc. IASTED Int. Conf. Parallel and Distributed Computing and Systems, MIT, USA, November 1999, pp. 320-328.

[17] A. K. L. Wong and W. Zhu, "A Multi-locking Mechanism on Shared Object DSM", Proc. $9^{\text {th }}$ Int. Conf. on Parallel and Distributed Systems, Taiwan, China, December 2002, pp. 91-96. [18] S. C. Woo, M. Ohara, E. Torrie, J. P. Singh, and A. Gupta, "The SPLASH-2 Programs: Characterization and Methodological Considerations", Proc. 22nd IEEE Annual Int. Symp. on Computer Architecture, NY, June 1995, pp. 24-36.

[19] W. Yu and A. Cox, "Java/DSM: A Platform for Heterogeneous Computing", Concurrency - Practice and Experience, Volume 9, Number 11, 1997, pp. 1213-1224. 